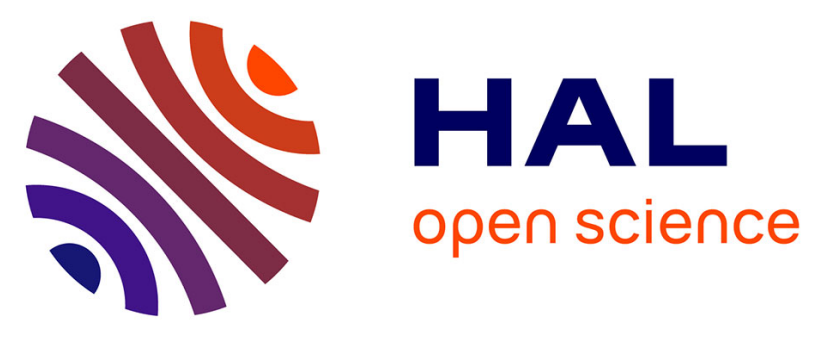

\title{
Que vit le méditant? Méthodes et enjeux d'une description micro-phénoménologique de l'expérience méditative
}

Claire Petitmengin, Martijn van Beek, Michel Bitbol, Jean-Michel Nissou, Andreas Roepstorff

\section{To cite this version:}

Claire Petitmengin, Martijn van Beek, Michel Bitbol, Jean-Michel Nissou, Andreas Roepstorff. Que vit le méditant? Méthodes et enjeux d'une description micro-phénoménologique de l'expérience méditative. Intellectica - La revue de l'Association pour la Recherche sur les sciences de la Cognition (ARCo), 2017, Les états modifiés de conscience en question: anciennes limites et nouvelles approches, 67, pp.219-242. hal-01653434

\section{HAL Id: hal-01653434 https://hal.science/hal-01653434}

Submitted on 1 Dec 2017

HAL is a multi-disciplinary open access archive for the deposit and dissemination of scientific research documents, whether they are published or not. The documents may come from teaching and research institutions in France or abroad, or from public or private research centers.
L'archive ouverte pluridisciplinaire HAL, est destinée au dépôt et à la diffusion de documents scientifiques de niveau recherche, publiés ou non, émanant des établissements d'enseignement et de recherche français ou étrangers, des laboratoires publics ou privés. 


\title{
Que vit le méditant?
}

\section{Méthodes et enjeux d'une description micro-phénoménologique de l'expérience méditative ${ }^{1}$}

\author{
Claire Petitmengin, Martijn van Beek, Michel Bitbol, Jean-Michel Nissou, Andreas Roepstorff
}

\section{Résumé}

Dans notre société où l'intérêt pour la méditation Bouddhiste connaît un engouement considérable, de nombreuses études sont maintenant menées sur les effets neurophysiologiques de la pratique méditative, et sur les corrélats neurophysiologiques des états méditatifs. Mais très peu d'études ont été conduites sur l'expérience de la pratique contemplative : ce que vit le méditant, instant après instant, aux différents stades de sa pratique, demeure presque invisible dans les sciences contemplatives contemporaines. Récemment, des méthodes d'entretien "microphénoménologiques" ont été développées pour nous aider à prendre conscience de notre expérience et à la décrire avec rigueur et précision. Cet article présente les résultats d'une étude pilote visant à appliquer ces méthodes à la description de l'expérience méditative.

La première partie de l'article décrit ces méthodes et leur adaptation à l'étude de l'expérience méditative. La seconde partie fournit des descriptions micro-phénoménologiques de deux processus dont la pratique permet au méditant de prendre conscience : le processus de perte de contact avec la situation présente et de génération d'une scène virtuelle dans les épisodes de "dérive attentionnelle", et le processus d'émergence d'une pensée. La troisième partie met en évidence l'intérêt de telles descriptions pour les pratiquants et pour les instructeurs de méditation, définit le statut de ces résultats, et propose des pistes de recherche.

Cet article ne décrit pas un état de conscience extraordinaire qui pourrait être induit par la pratique méditative, mais l'état de conscience modifié très ordinaire consistant à s'absenter de la situation présente pour s'absorber dans une situation virtuelle. Il propose une méthode permettant de produire une description "micro-phénoménologique" de la dynamique d'émergence et de résorption d'un état de conscience modifié.

\section{Introduction}

Dans notre société où l'intérêt pour la méditation, notamment pour ses formes sécularisées comme la pratique de la "pleine conscience", se développe de manière exponentielle, de nombreuses études sont maintenant menées sur les effets neurophysiologiques de la pratique méditative (e.g. Lazar et al., 2006; Farb et al., 2007; Slagter et al., 2007; Grossman et al., 2007; Grant et al., 2010; Holzel et al., 2011; Desbordes et al., 2012; Jensen et al., 2012; Allen et al., 2012), et sur les corrélats neurophysiologiques des états méditatifs (e.g. Brefczynski-Lewis et al. 2007; Brewer et al., 2011; Lutz et al., 2008; Lutz et al., 2013; van Leeuwen et al., 2012; Mrazek et al., 2013; Reiner et al;, 2013; Zanesco et al., 2013), et fournissent des résultats importants. Par exemple, il a été montré que la pratique de la méditation peut réduire le stress et le taux de rechute

1 Cet article est la traduction de l'article "What is it like to meditate? Methods and issues for a microphenomenological description of meditative experience" à paraitre dans le Journal of Consciousness Studies.

Le projet dit "Phenopilot" a été financé par une subvention de l'Institut Mind and Life. Les travaux réalisés au Danemark ont été partiellement financés par des subventions de la Fondation Velux et du Danish Research Council for Culture and Communication. 
dépressive (Teasdale et al., 2000; Segal et al., 2002; Kuyken et al. 2015). Mais très peu d'études ont été conduites sur l'expérience vécue de la pratique contemplative : ce que vit le méditant, instant après instant, aux différents stades de sa pratique, a été peu étudié par les sciences contemplatives contemporaines (e.g. Khalsa et al., 2008; Fox et al., 2012; Ataria, 2014, 2015). Au mieux, les études neurophysiologiques de la méditation accordent un statut auxiliaire à quelques descriptions grossières de classes d'expériences méditatives (Lutz et al., 2012). Même l'étude récente qui utilise une matrice de catégories phénoménologiques comme outil heuristique pour générer des hypothèses sur les corrélats neuronaux des pratiques méditatives (Lutz et al., 2015), dérive ces catégories de l'analyse de textes et de manuels, pas d'une description phénoménologique de ces pratiques.

Ce déficit pourrait s'expliquer par l'hypothèse répandue selon laquelle, puisque l'expérience méditative - comme toute expérience - est produite par l'activité cérébrale, la connaissance de ses corrélats neuronaux est suffisante pour comprendre ce qui se joue dans la méditation et comprendre ses effets. Il pourrait aussi s'expliquer par le discrédit qui frappe l'introspection dans les sciences occidentales qui, depuis Auguste Comte arguant qu'il est impossible de marcher dans la rue tout en se regardant marcher depuis le balcon, se sont construites sur son exclusion. De plus, dans les traditions contemplatives, il n'est pas d'usage de parler de son expérience, excepté avec son enseignant. Un participant à notre étude, pratiquant de la tradition Dzogchen du Bouddhisme Tibétain depuis 45 ans, a remarqué après le deuxième entretien : "C'est nouveau pour moi, je ne suis pas habitué. Je n'ai jamais eu l'occasion de partager mes expériences avec qui que ce soit, même avec mes enseignants, même avec ma femme." (Alain)

Notre étude est née de l'hypothèse que le manque de connaissance de l'expérience méditative bloque à la fois la compréhension des effets de la pratique et celle de ses corrélats : de ses effets, car seule une description fine de l'expérience vécue du sujet permettrait de comprendre les processus mobilisés pendant la méditation, qui pourraient expliquer ces effets (Philippot \& Segal, 2009); de ses corrélats, car plus les techniques de neuro-imagerie cérébrale s'affinent, plus l'interprétation des données qui en sont issues demande une description précise de l'expérience vécue des sujets dont on enregistre l'activité (Lachaux, 2011). De telles descriptions sont maintenant rendues possibles par le développement de méthodes "micro-phénoménologiques" qui nous permettent de prendre conscience de notre expérience et de la décrire avec rigueur et précision.

Cet article décrit les résultats d'un projet pilote visant à appliquer ces méthodes à la description de l'expérience méditative. La première partie de l'article décrit ces méthodes et leur ajustement à l'étude de l'expérience méditative. La seconde partie fournit les descriptions microphénoménologiques de deux processus dont la pratique permet au méditant de prendre conscience : le processus de perte de contact avec la situation présente et de génération d'une scène virtuelle dans les épisodes de "dérive attentionnelle", et le processus d'émergence d'une pensée. La troisième partie met en évidence l'intérêt de telles descriptions pour les pratiquants et pour les instructeurs de méditation, définit le statut de ces résultats, et propose des pistes de recherche.

Cet article ne décrit donc pas un état de conscience extraordinaire qui pourrait être induit par la pratique méditative, mais l'état de conscience modifié très ordinaire consistant à s'absenter de la situation présente pour s'absorber dans une situation virtuelle. Il propose une méthode permettant de produire une description "micro-phénoménologique" de la dynamique d'émergence et de résorption d'un état de conscience modifié. 


\section{Méthode et objectifs}

\section{La Micro-phénoménologie}

La micro-phénoménologie est une méthode de phénoménologie descriptive inspirée de "l'entretien d'explicitation" initialement développé par Pierre Vermersch (1994/2014, 2009, 2012) pour aider des personnes engagées dans des pratiques professionnelles à prendre conscience de la part implicite de leurs actions physiques et mentales. Cette méthode d'entretien a ensuite été adaptée à la recherche en sciences cognitives pour décrire l'expérience associée à tout processus cognitif, y compris des processus impliquant le corps comme la perception ou l'émotion (Petitmengin 2006). Elle a été complétée par une méthode permettant d'analyser les rapports verbaux et d'y détecter des régularités sous la forme de structures génériques (Petitmengin, 1999; Petitmengin et al., soumis), et par des méthodes permettant d'évaluer la fiabilité des rapports et de valider ces résultats (Petitmengin \& Bitbol, 2009; Petitmengin et al., 2013; Bitbol \& Petitmengin, 2013, 2016). Cette méthode permet au chercheur de recueillir des descriptions d'un niveau de fiabilité élevé et d'un degré de granularité fin de la microdynamique d'expériences singulières, jusque dans leur dimension pré-réfléchie.

Une part importante de notre expérience demeure non reconnue, non remarquée, "pré-réfléchie" dans le langage phénoménologique. Ceci est dû au fait que notre attention est presque totalement absorbée dans le contenu, le "quoi" de notre activité, au détriment de cette activité elle-même, c'est-à-dire du "comment". Nous sommes un peu comme des personnes aveugles explorant des objets du bout de leur canne, dont l'attention est entièrement dirigée vers ces objets, mais qui ne sont pas réflexivement conscients du contact et des variations de pression de la canne dans la paume de leurs mains. Comme ces personnes aveugles, nous utilisons ces informations pour agir, mais elles demeurent largement non remarquées. L'objectif d'un entretien microphénoménologique est d'aider les sujets interviewés à réorienter leur attention du contenu de leur expérience vers le mode et la dynamique d'apparition de ce contenu, et de les décrire avec précision. Cette redirection est comparable au geste de réduction phénoménologique décrit par Edmond Husserl: il s'agit d'extraire notre attention de sa focalisation exclusive sur les objets, et d'élargir le champ attentionnel (Bitbol, 2014) de manière à révéler et décrire la "vie intentionnelle" sous-jacente de la conscience, et encore plus profondément, le niveau pré-intentionnel de "l'autoaffection de la vie" (Henry, 2000).

Pour effectuer cette réorientation de l'attention il est tout d'abord nécessaire d'amener la personne interviewée à décrire une expérience singulière. Si vous lui demandez : "'Quelle est ton expérience lorsque tu médites ?", il est à peu près certain que vous obtiendrez une description générale, correspondant aux instructions de méditation qu'elle a reçues, ou à la représentation qu'elle se fait de son expérience. Votre seule chance d'obtenir une description plus conforme à ce qu'elle vit réellement est 1) de la guider vers la description d'un moment particulier d'expérience, situé dans l'espace et dans le temps; 2) de la ramener vers l'expérience concrète et singulière qu'elle est en train de décrire, à chaque fois qu'elle s'en écarte pour exprimer des commentaires, des justifications, des explications et des croyances - qui ne correspondent à ce qu'elle vit mais à ce qu'elle pense, imagine, ou croit vivre. Par exemple, à chaque fois que le sujet utilise un terme abstrait, l'intervieweur peut l'inviter à décrire l'action concrète qui sous-tend ce terme, en reprenant ce dernier sous la forme interrogative. Si le sujet interviewé dit : "J'ai adopté un mode d'attention ouvert", l'intervieweur peut ainsi répondre : "Prenez le temps de retrouver ce moment où vous avez adopté un mode d'attention ouvert... A ce moment, comment vous y prenez-vous pour ouvrir votre attention ?". Le ralentissement du débit verbal, la présence d'hésitations et de silences, de gestes 
coverbaux, l'utilisation du pronom "je" et de verbes d'action, sont alors les indices que le sujet n'est pas en train de réciter des connaissances toutes faites, mais de découvrir des processus préréfléchis.

Dans la plupart des cas, comme il est difficile de décrire une expérience pendant qu'elle se déroule, il existe un écart temporel entre l'expérience initiale et sa description. La deuxième clé de l'entretien micro-phénoménologique consiste donc à aider le sujet à rejouer ou "évoquer" l'expérience, qu'elle soit juste passée ou plus éloignée dans le temps, en retrouvant précisément son contexte spatio-temporel, puis les sensations visuelles, auditives, tactiles, kinesthésiques et éventuellement olfactives associées au début de l'expérience à décrire. Le sujet "évoque" ce moment lorsqu'il le retrouve, au point que la situation passée devient plus présente pour lui que la situation actuelle. L'intensité de l'évocation peut être vérifiée grâce à des indices objectifs comme l'utilisation du temps présent et le décrochage du regard. L'expérience évoquée peut être "invoquée" si elle s'est produite dans le passé indépendamment de l'entretien, ou "provoquée" pour les besoins de l'entretien, ordinairement juste avant (Vermersch 1994/2010). Dans la présente étude, nous avons parfois amené des participants à décrire des moments significatifs d'expériences méditatives passées. Mais étant donnée la subtilité de l'expérience méditative, et la difficulté d'évoquer ce type d'expérience dans ses détails les plus fins, nous nous sommes plutôt concentrés sur des expériences provoquées, en introduisant deux variantes :

- Entretien interrompu par une session de méditation: pendant l'entretien, il est suggéré au méditant de méditer pour trouver la réponse à une question donnée.

- Pouvez-vous essayer de décrire plus précisément ce que vous faites au moment où vous réorientez votre attention?

[Pause pendant laquelle Helen pratique]

- Je vais essayer de l'expliquer. Maintenant quand je suis assise et que je ressens cette activité, j'ai l'impression qu'elle se passe par ici.

- Méditation interrompue par l'entretien: l'entretien débute après interruption de la méditation à un instant aléatoire, par le son d'un gong par exemple, et porte sur le moment précis ayant précédé l'interruption.

La troisième clé de l'entretien consiste à aider le sujet à relâcher la focalisation de l'attention sur le contenu, le "quoi" de l'expérience, de manière à laisser apparaître le "comment". Par exemple, l'intervieweur aide le sujet à réorienter son attention du contenu d'une image mentale qui apparaît à sa conscience, vers la dynamique d'apparition, la genèse de ce contenu : les phases très rapides qui précèdent sa stabilisation, et pour chaque phase les subtils micro-gestes internes réalisés pour susciter, reconnaître, évaluer, compléter ou éventuellement écarter l'image. Pour recueillir une telle description diachronique, l'art de l'intervieweur consiste à poser des questions qui attirent l'attention du sujet sur les différents moments de l'expérience, sans suggérer de contenu, par exemple : "par quoi avez-vous commencé ? ", "que s'est-il passé juste après ?", "quand vous avez fait ceci, qu'avez-vous fait ?", "quand vous avez ressenti ceci, qu'avez-vous ressenti ?". Ce questionnement "vide de contenu" lui permet d'obtenir une description précise sans infiltrer ses propres présupposés ou créer de "faux souvenirs".

- Quand j'ai réalisé que j'étais partie, la pensée s'est évanouie.

- Comment s'est-elle évanouie ? Etait-ce instantané ou progressif ?

- C'était très rapide, mais cela a quand même pris un moment.

- Et qu'est-il arrivé pendant ce moment ?

- J'ai relâché, j'ai relâché ma tension sur cette pensée. 
- Et quand tu as relâché ta tension sur cette pensée, qu'as-tu relâché ?

- En fait j'ai relâché une légère tension dans ma tête

- Où exactement était cette légère tension dans ta tête ?

- Elle était en haut, sur la droite et à l'avant de ma tête.

- Et quand tu l'as relâchée, comment t'y es-tu prise, qu'as-tu fait ?

- Et ainsi de suite. (Lise, 20 years of practice)

La structure d'un entretien est une structure itérative consistant à amener le sujet à évoquer l'expérience à plusieurs reprises, tout en guidant son attention vers une maille diachronique et synchronique de plus en plus fine à chaque passage, jusqu'à ce que le degré de précision souhaité soit atteint. La description de quelques secondes d'expérience prend environ une heure. La cohérence de la description, malgré la structure itérative de l'entretien, est un critère complémentaire d'authenticité convaincant.

\section{Objectifs et organisation de l'étude}

La méthode micro-phénoménologique nous avait permis de recueillir la description très détaillée d'expériences réputées inaccessibles à la conscience ou difficilement descriptibles, comme la microgenèse d'une "intuition" (Petitmengin, 1999, 2007; Remillieux, 2014), l'émergence d'une crise d'épilepsie (Petitmengin et al., 2006; Petitmengin, 2010), la microgenèse d'un choix (Petitmengin et al., 2013), ou l'émergence de la fameuse "illusion de la main en caoutchouc" (Valenzuela et al., 2013). Encouragés par ces résultats, nous avons mené une étude pilote de description d'expériences méditatives vécues dans le contexte de la tradition Bouddhiste.

Le terme Sanskrit ordinairement traduit par "méditation" est bhāvanā, littéralement "entraînement". Méditer, c'est s'entraîner à "voir les choses comme elles sont" ${ }^{2}$. Les techniques de méditation Bouddhistes peuvent être divisées en deux groupes : śamatha et vipaśyanā, pour utiliser les termes sanskrits. Bien que ces termes puissent désigner différentes pratiques dans les différentes écoles Bouddhistes, śamatha, la pratique du "calme mental", vise ordinairement à apaiser le flot des pensées par la concentration de l'esprit sur un unique objet intérieur ou extérieur. Vipaśyanā, la "vision pénétrante", consiste à appliquer l'attention stabilisée par śamatha à l'ensemble de l'expérience : les sensations corporelles, les émotions, les pensées, de manière à "voir les choses comme elles sont" ${ }^{3}$.

Nous avons interviewé 13 pratiquants ayant 5 à 45 années d'expérience de telles pratiques méditatives, soit 4 hommes et 9 femmes âgés de 28 à 62 ans : 5 italiens, 3 français, 3 danois, un norvégien et un anglais, interviewés dans leur langue maternelle. Les extraits d'entretiens présentés ici ont été choisis en fonction de leur degré de précision et de leur pertinence pour illustrer les deux processus méditatifs sur lesquels se concentre le présent article (ils ne sont donc pas nécessairement représentatifs de l'ensemble de l'expérience méditative des participants). Ces extraits ont ensuite été traduits en français si nécessaire. Afin de protéger l'anonymat des participants, nous avons utilisé des pseudonymes.

En bref, le projet visait à répondre aux deux questions suivantes : est-il possible de considérer l'expérience méditative comme un objet de recherche à part entière, sans la réduire à ses corrélats neurophysiologiques ? Si oui, pourquoi et pour qui est-il intéressant de la faire ? Le but du projet n'était pas de comparer les pratiques spécifiques des différents méditants, mais plus modestement:

\footnotetext{
${ }^{2}$ See eg. Tulku Urgyen Rinpoche, As It Is, vol. 1 \& $2 .$.

${ }^{3}$ For typologies of Buddhist contemplative practices, see, e.g., Germano and Hillis 2005; Lutz et al. 2007; Lutz et al. 2015.
} 
1. de vérifier si les techniques micro-phénoménologiques permettent de recueillir des descriptions précises et fiables d'expériences méditatives;

2. d'évaluer l'utilité de telles descriptions pour le pratiquant et pour l'instructeur de méditation;

3. d'évaluer les besoins d'adaptation de ces techniques à ce domaine d'étude, et de développer une méthode de recherche utilisable par de futurs projets;

4. d'identifier des directions de recherche future sur l'expérience méditative.

Chaque méditant a participé à deux ou trois entretiens. Le "protocole expérientiel" du premier entretien était le suivant :

1. Pré-entretien: on explique le contexte et les objectifs du projet de recherche au pratiquant; des détails lui sont demandés sur sa pratique : tradition, pratique usuelle, nombre d'années et d'heures cumulées de pratique, fréquence de pratique. S'il ne connaît pas la méthode d'entretien, on lui propose de vivre un petit entretien micro-phénoménologique.

2. Session de méditation (20 minutes) : on demande au pratiquant de pratiquer śamatha, c'est-àdire de concentrer son attention sur sa respiration, et quand il ou elle réalise que son attention a quitté la respiration, de revenir à la respiration.

3. Entretien micro-phénoménologique (60 à 90 minutes) : après une description globale du déroulement de la session, l'entretien se focalise sur un ou deux moments spécifiques.

4. Post-entretien : on pose au pratiquant des questions sur l'entretien lui-même, comme: "L'entretien t'a-t-il permis de prendre conscience de quelque chose ? As-tu appris quelque chose pendant l'entretien ?".

Le "protocole expérientiel" du deuxième entretien (et éventuellement du troisième) est le suivant :

1. Pré-entretien: après un bref rappel de la recherche, on demande au participant de décrire les effets éventuels du premier entretien sur sa pratique entre les deux entretiens.

2. Session de méditation (20-30 minutes): le participant fait sa pratique habituelle.

3. Entretien micro-phénoménologique comme dans la session 1.

4. Post-entretien comme dans la session 1.

\section{Exemples de pistes d'investigation de l'expérience méditative}

Cette seconde partie est consacrée aux descriptions micro-phénoménologiques de deux processus dont la pratique méditative permet au pratiquant de prendre conscience.

a) Le premier est le double processus de perte de contact avec la situation présente et de génération d'une situation virtuelle dans les épisodes de "dérive attentionnelle", suivi du double processus inverse de disparition de la situation virtuelle et de retour à la situation présente et de maintien en contact avec elle, permis par la pratique méditative.

b) Le second est celui de l'émergence d'une pensée.

\subsection{Dérive attentionnelle}

Le but de la pratique méditative du calme mental n'est pas de prendre conscience du déroulement des épisodes de dérive, mais de stabiliser l'attention en la ramenant régulièrement sur le support de concentration. Les entretiens micro-phénoménologiques permettant d'explorer tout ce qui se passe pendant une session de méditation, ils nous ont permis de décrire non seulement l'acte intérieur consistant à ramener l'attention sur la respiration et l'effet de cet acte, mais aussi ce qui 
arrive lorsque l'attention a quitté son support, autrement dit le processus très ordinaire mais très peu connu d'un point de vue phénoménologique, nommé "dérive attentionnelle" (en anglais "mindwandering").

\section{Contexte scientifique}

Bien que ce phénomène ait auparavant échappé à la recherche scientifique (sauf Schooler, 2005; Smallwood \& Schooler, 2006), le nombre de publications sur la dérive attentionnelle a considérablement augmenté depuis qu'une étude publiée dans Science a montré que nous passons plus de la moitié du temps perdu dans des scènes virtuelles passées ou futures, sans même être conscient d'avoir perdu le contact avec la réalité présente (Killingsworth \& Gilbert, 2010). C'est le cas même lorsque nous sommes engagés dans des activités difficiles ou à risque comme la conduite automobile (Galera et al., 2012; Yanko \& Spalek, 2013).

La méthode la plus utilisée pour étudier l'expérience de dérive attentionnelle consiste à fournir au sujet un petit appareil qui émet un "bip" à intervalles aléatoires. Quand le bip se produit, il doit noter si son esprit vagabondait ou non juste avant le bip, et répondre à un questionnaire. Ces études ont permis d'étudier le contenu de la dérive (focus temporel, valence émotionnelle) et ses résultats, et de rechercher une relation éventuelle entre les deux (Smallwood \& Andrews-Hanna, 2013; Andrews-Hanna et al., 2013; Ruby et al., 2013a). Par exemple elles ont permis d'établir une corrélation entre dérive attentionnelle et sentiment de mal-être (Smallwood et al., 2007; Killingsworth and Gilbert, 2010). Mais les méthodes utilisées ne donnent pas accès au processus de dérive attentionnelle, au déroulement des épisodes de dérive, qui permettrait de mieux comprendre ces effets, qui restent inexpliqués (Mooneyham and Schooler, 2013; Schooler et al., 2014).

\section{Exemples de descriptions et d'analyses}

Nos micro-phénoménologiques nous ont permis de recueillir des descriptions de ce déroulement, qui prend la forme :

- d'un double processus de perte de contact avec la situation présente et de génération d'une situation virtuelle,

- suivi du double processus inverse de disparition de la situation virtuelle et de retour à la situation présente.

Nous avons tenté d'aller plus loin dans la description de ces processus. Voici un exemple de description qui bien que triviale n'est pas dénuée d'intérêt.

\section{Extrait 1: Processus de generation d'une scène virtuelle}

"Une pensée se produit, qui met en scène plusieurs personnages. C'est comme une sorte d'espace qui s'ouvre, le mot "bulle" est bien adapté. Une sorte de bulle qui part de ma tête, devant, en haut et à gauche. Le volume de cette bulle est entretenu par mon discours interne, un peu comme la lumière est entretenue par le moteur d'une dynamo et varie avec les variations de régime du moteur. Je vois les personnages comme par mes yeux, mais je n'emmène pas mon corps dans l'histoire. C'est-à-dire que je ne suis plus que des yeux, mon corps n'est pas dans la scène. En même temps, cette scène suscite beaucoup de sensations physiques en moi, par exemple au niveau de la poitrine, mais (pendant la dérive) je n'en suis pas consciente, j'ai perdu le contact avec elles. C'est comme si ma tête était coupée de mon corps." (Lise, 20 ans de pratique)

L'intérêt de cette description est double : 1) elle montre qu'il est possible de se souvenir d'un épisode de dérive, qui a été vécu sans conscience d'être vécu, autrement dit de manière pré- 
réfléchie ou non reconnue; 2) elle nous permet de commencer à identifier les paramètres du processus de construction d'une scène virtuelle, qui sont indépendants du contenu de la scène :

- Le lieu d'émergence de la scène virtuelle dans "l'espace vécu" du sujet:

"C'est comme une sorte d'espace qui s'ouvre, le mot "bulle" est bien adapté. Une sorte de bulle qui part de ma tête, devant, en haut et à gauche."

- Les modalités sensorielles impliquées dans la scène, ici les modalités auditive "discours interne", visuelle : "je vois les personnages", et intéroceptive " beaucoup de sensations physiques en moi".

- Le degré et mode de conscience des sensations suscitées par la scène virtuelle. Au moment de l'épisode de dérive, Lise semble consciente de son expérience visuelle : "je vois les personnages", tout en étant en un sens inconsciente de cette expérience parce qu'elle est alors absorbée dans la scène virtuelle et inconsciente que son attention dérive (si elle l'avait réalisé, elle aurait en effet ramené son attention sur sa respiration). Nous avons décidé de nommer ce mode particulier d'absence de conscience "l'inconscience de dérive". Au même moment, Lise n'est pas consciente de ses sensations corporelles : "je n'en suis pas consciente, j'ai perdu le contact avec elles. C'est comme si ma tête était coupée de mon corps". Elle est donc en un sens doublement non consciente de ses sensations corporelles : non consciente parce qu'inconsciente que son attention dérive, mais aussi non consciente d'avoir des sensations corporelles ${ }^{4}$.

- Les positions perceptuelles du sujet dans la scène virtuelle, qui sont ici une position en première personne (ou égocentrique) dans la modalité visuelle : "Je vois les personnages comme par mes yeux"; et une absence de position perceptuelle (a "no-body" position) dans la modalité intéroceptive : "je ne suis plus que des yeux, mon corps n'est pas dans la scène".

- Le "mode d'entretien" de la scène virtuelle : "Le volume de cette bulle est entretenu par mon discours interne, un peu comme la lumière est entretenue par le moteur d'une dynamo et varie avec les variations de régime du moteur."

L'extrait suivant, issu d'un autre interview, met en évidence un mode d'entretien de la scène différent, et nous permet de compléter notre inventaire des paramètres du processus de dérive.

\section{Extrait 2: Processus de génération d'une scène virtuelle et perte de contact avec la situation actuelle}

"Je me perds dans une pensée consistant à imaginer que je suis en train de couper en morceaux, puis de faire une purée avec le potimarron qui est en train de cuire, là pendant que je médite. Je ne me dis rien à moi-même, je fais intérieurement le geste d'écraser la purée avec une fourchette.

Je ressens très bien (maintenant pendant l'entretien) les gestes, l'effort dans mes bras. Pendant ce temps (pendant la méditation), je perds contact avec mon corps ici et maintenant : sa position, le contact de mes jambes avec le sol, mes mains sur les genoux, mes sensations internes. Je ne suis pas non plus vraiment consciente des sensations suscitées par la scène imaginaire... la sensation d'effort dans les bras.

J'en deviens consciente rétrospectivement, quand je suis revenue, pendant que je garde encore la trace de ce moment." (Lise)

- Mode d'entretie de la scène. Dans cette expérience, la scène virtuelle est générée et entretenue grâce à des gestes qui sont anticipés intérieurement : "je suis en train de couper, puis de faire une

\footnotetext{
${ }^{4}$ See Petitmengin 2006, p. 234
} 
purée avec le potimarron", "je fais intérieurement le geste d'écraser la purée avec une fourchette". Il est intéressant de noter que dans les deux extraits précédents, la scène virtuelle est entretenue par des micro-gestes subtils - dans le premier cas des gestes laryngo-buccaux suscités par le discours interne, dans le second cas des micro-gestes consistant à mimer intérieurement un mouvement mobilisant le haut du corps.

Dans le deuxième extrait, le sujet différencie deux types de sensations corporelles pendant l'épisode de dérive : les sensations associées à la situation actuelle, et les sensations suscitées par la scène virtuelle.

- Degré et mode de conscience des sensations corporelles actuelles. "Je perds contact avec mon corps ici et maintenant : sa position, le contact de mes jambes avec le sol, mes mains sur les genoux, mes sensations internes."

- Degré de conscience des sensations suscitées par la scène virtuelle. "Je ne suis pas non plus vraiment consciente des sensations suscitées par la scène imaginaire... la sensation d'effort dans les bras." (Double inconscience)

- Une nouvelle catégorie descriptive apparaît, que nous appelons la persistance de la scène virtuelle : "pendant que je garde encore la trace de ce moment". Après que Lise ait réalisé qu'elle s'était absentée, cette "trace" ou persistance lui permet d'évoquer la scène virtuelle et de la décrire. La disparition de "l'inconscience de dérive" pendant l'évocation lui permet de décrire les sensations qui étaient présentes au moment de l'épisode, et cette "trace" lui permet de devenir réflexivement consciente de sensations dont elle était "doublement inconsciente" à cet instant : "J'en deviens consciente rétrospectivement, quand je suis revenue, pendant que je garde encore la trace de ce moment."

\section{Extraits 3 et 4: Processus de reprise de contact avec la situation actuelle}

"Quand je reviens au niveau des narines, il y a quelque chose qui se détend dans toute ma tête. Avant il y avait une petite crispation, légère, au niveau du crâne, à l'intérieur, dont je n'avais pas conscience. Et quand je reviens, à la première inspir, ça s'aère. Ca se soulage. Cette tension, qui n'en est pas vraiment une, c'est très léger, avec un poids si tu veux, avec une densité et un poids, ça se libère, ça se dilue." (Paul, 20 ans de pratique)

"Quand je rentre en contact avec les sensations physiques suscitées par cette scène (virtuelle), ça suscite un sentiment particulier d'apaisement, de me retrouver moi-même, qui s'accompagne d'un profond soupir." (Lise)

Les extraits 3 et 4 mettent en évidence certains paramètres du processus inverse de reprise de contact avec la situation actuelle :

- l'effet suscité par le retour aux sensations corporelles actuelles. La réorientation de l'attention vers la sensation de la respiration : "quand je reviens au niveau des narines", "à la première inspir", suscite un sentiment de soulagement and d'apaisement : "Quelque chose se détend dans toute ma tête", "Ça se soulage", "Ça se libère, ça se dilue".

- la présence d'une subtile sensation corporelle qui était présente au moment de l'épisode de dérive (et peut-être suscitée par lui) : " Avant il y avait une petite crispation, légère, au niveau du crâne, à l'intérieur".

- L'absence de conscience de cette sensation au moment de l'épisode (Double inconscience) : "Avant il y avait une petite crispation (...) dont je n'avais pas conscience". 
- Le déclencheur de la prise de conscience de cette sensation : l'extrait suggère que c'est le mouvement même de relâchement de la crispation (lui-même suscité par la réorientation de l'attention vers les narines), qui permet au sujet de prendre conscience a) de la crispation qui est relâchée, et b) du fait que cette crispation était déjà présente pendant que l'attention avait quitté les narines.

- l'effet du retour aux sensations corporelles suscitées par la scène virtuelle, qui est aussi un sentiment de soulagement : "un sentiment particulier d'apaisement, de me retrouver moi-même, qui s'accompagne d'un profond soupir".

\subsection{Les phases initiales de l'émergence d'une pensée}

Quatre pratiquants (Lise, Paul, Anna and Helen) ont décrit en détails les premières phases de l'émergence d'une pensée, avant l'apparition d'une scène virtuelle. Leurs témoignages s'accordent sur le processus micro-dynamique suivant.

\section{1) Une micro-implusion}

\section{- Forme sensorielle de la pensée naissante}

"La 'pensée' apparaît comme une minuscule crispation." (Lise)

"Un petit élan ici (il montre le centre de sa poitrine). Il est associé à la respiration, à l'inspiration." (Paul)

"Ce n'est pas tant une image, qu'un ressenti que quelque chose est en train d'émerger. Comme un petit mouvement... une perturbation. Ce n'est pas encore une pensée. C'est juste une sorte d'animation. Quelque chose se prépare." (Anna, 12 ans de pratique)

\section{- Lieu d'émergence}

"Ici (il montre le centre de sa poitrine)." (Paul)

\section{2) Une tension vers ce premier mouvement}

"Il se produit une sorte de mouvement vers ce mouvement, si l'on peut dire." (Anna)

\section{- Localisation de la tension : les yeux et la tête}

"Le regard se fixe, et il y a presque comme une tension dans les yeux. Bien que je ne regarde rien de particulier, le sens visuel est mobilisé comme si je regardais." (Anna)

"Une tension correspondant à l'intention de faire quelque chose, semblable au mouvement de scruter visuellement, de faire un effort pour mieux voir quelque chose au loin, qui donne une sensation de tension dans le crâne." (Anna)

\section{- Origine de la tension: le fait de retenir la respiration, serrer la gorge}

"C'est comme si... retenir sa respiration, la gorge se serre... Un sentiment de curiosité et d'être à l'arrêt et d'aller vers quelque chose... de retenir sa respiration aussi. Cela se produit au niveau de la gorge. Le regard... le deuxième mouvement... le resserrement vient d'ici, plutôt que de la tête." (Anna)

\section{- Effet de la tension}

"Alors la contraction dans la tête se produit, et on a comme l'impression que la tête est coupée du corps, au niveau de la gorge. Le flux d'énergie entre le corps et la tête se coupe et s'accumule dans la gorge. C'est inconfortable, donc ça attire l'attention." (Anna)

"Il ne s'agit pas de quelque chose de massif, c'est vraiment minime. Une sorte... d'engourdissement subtil, oui. C'est comme si la vie ne circulait pas complètement librement dans le corps. Parce que l'attention, c'est comme si elle était engourdie, et ça donne un sentiment, une sorte d'inconfort. Ce n'est 
pas comme d'avoir la nausée, mais c'est du même genre. Il y a moins de contact. Moins d'animation par la vie." (Helen, 20 ans de pratique)

Les deux extraits ci-dessus mettent en évidence :

Un manque de circulation du flux d'énergie dans le corps: " Le flux d'énergie entre le corps et la tête se coupe et s'accumule dans la gorge"; "C'est comme si la vie ne circulait pas complètement librement dans le corps." "Moins d'animation par la vie".

Une impression de déconnection de la tête et du corps : "l'impression que la tête est coupée du corps".

Le point de déconnexion : " Le flux d'énergie entre le corps et la tête s'accumule dans la gorge"

Un sentiment subtil d'inconfort suscité par cette déconnexion : "C'est inconfortable"; "Une sorte... d'engourdissement subtil"; "un sentiment, une sorte d'inconfort"; "Ce n'est pas comme d'avoir la nausée, mais c'est du même genre".

\section{3) Le relâchement de la tension}

- Le déclencheur du relâchement est pour Anna la prise de conscience du sentiment de déconnection, elle-même déclenchée par la sensation d'inconfort associée à cette déconnection :

"C'est inconfortable, donc ça attire l'attention. C'est comme ça que je sais que la déconnection s'est produite." (Anna)

- Le relâchement de la tension peut être spontané, involontaire :

"C'est comme si ça se relâchait de soi-même... avec la conscience que ça se resserre. C'est juste... en prendre conscience suffit à le relâcher." (Anna)

- Mais il est aussi décrit comme facilité par des micro-gestes intérieurs spontanés :

Relâcher la respiration, la contraction dans la tête et dans la gorge

"Je relâche la respiration; je relâche la contraction si elle est dans la tête ou dans la gorge. Je relâche toute forme de fixation; juste laisser les choses redevenir fluides. Et les laisser entrer et sortir librement." (Anna)

\section{Utilisation de la vision périphérique}

"Il y a presque comme une tension dans les yeux aussi... comme une fixation visuelle sur quelque chose. Donc juste la détendre dans un champ visuel plus panoramique." (Anna)

"Normalement, prendre conscience qu'il y a une fixation de tension est suffisant. Mais si j'ai besoin d'utiliser plus de procédés, alors j'utilise habituellement la conscience visuelle, en laissant ma vision périphérique s'élargir à plus de 180 degrés. Et cela relâche aussi la fixation dans les yeux et dans la tête." (Anna)

\section{Conscience, densification du bas et de l'arrière du corps}

"Etre plus conscient de l'arrière de mon corps, plutôt que seulement de ce qui est devant seulement." (Anna) 
"Je descends dans mon corps. Je démobilise la tête pour descendre dans mon corps. Cela se densifie dans le bas du corps." (Lise)

Dans l'extrait suivant, l'interviewer aide Lise à décrire comment elle fait pour "descendre dans son corps".

- A ce moment de la méditation je descends dans mon corps.

- Comment fais-tu pour descendre dans ton corps?

- Je vais vers l'arrière de ma tête, c'est-à-dire que je déplace mon centre de gravité de l'avant de ma tête vers l'arrière de ma tête.

- De quel centre de gravité tu parles?

- Mon attention

- Le centre de gravité de ton attention?

- Oui voilà. Qui est plutôt, comme souvent, au niveau de la tête, du centre de ma tête, juste derrière les yeux. En fait je le déplace plus vers l'arrière de la tête.

- Comment tu fais pour le déplacer?

- J'appuie un petit peu avec mes paupières sur les yeux. Et en faisant ça c'est comme si je démobilisais le regard, je démobilise cette zone. Je sens plus l'arrière de mon crâne, qui devient plus dense. Et en même temps, rapidement, cette densité elle descend dans tout l'arrière du corps, le long de la colonne vertébrale.

- Quand cette densité descend le long de la colonne vertébrale, qu'est-ce qui descend?

- Je sens vraiment un petit courant qui descend le long de la colonne vertébrale, c'est comme un petit fourmillement, c'est agréable, cette zone autour de la colonne vertébrale qui se mobilise, et qui se densifie. Et assez rapidement, j'ai un grand soupir... ça soulage. C'est comme si mes épaules, ma colonne vertébrale, mes reins, redevenaient vivants, se remettaient à s'animer, à sentir. Et quand le soupir arrive, le bien être s'étend au niveau de la poitrine, qui s'aère, qui s'ouvre. La respiration réanime aussi le devant du corps.

- [...] Quand je rouvre les yeux, je ne regarde plus avec mes yeux, je regarde depuis l'arrière de ma tête.

- Quelle conséquence ça a sur le regard, sur ton champ de vision ?

- C'est une autre manière de regarder, d'appréhender, que visuelle. Ce n'est plus seulement visuel, c'est quelque chose de plus vaste que le seulement visuel. C'est comme si je regardais avec l'arrière de mon corps.

- Comment fais-tu pour regarder avec l'arrière de ton corps ?

- C'est plus du tout le même regard. C'est un regard qui est une sorte de ressenti de l'espace. Je ne suis plus focalisée sur un objet avec le devant de la tête et des yeux, c'est l'arrière de ma tête qui regarde donc c'est beaucoup plus vaste. Le champ est ouvert au maximum. Il y a moins de relief en fait. Disons qu'il n'y a pas des objets qui se détachent, c'est plus plat, dans un sens, en tout cas à ce moment là. A ce moment là c'est plus en deux dimensions qu'en trois dimensions.

\section{- Effets du relâchement de la tension}

\section{Disparition de la pensée naissante}

"Quand je relâche la tension, la pensée s'évanouit." (Lise)

\section{Reconnection de la tête et du corps}

"On a l'impression que l'attention (awareness) s'ouvre à nouveau. Et le flux entre la tête et le corps... on a l'impression qu'il y a de plus de rencontre, plus de connexion, entre la tête et le corps." (Anna)

Sensation de flux et de chaleur, conscience des sensations corporelles, connexion entre moi et le monde 
"C'est chaud. Comme si le sang coulait librement, chaleur, impression d'être centré, sensations corporelles du corps. Je peux sentir l'inspiration, l'expiration, au niveau des cellules. Je peux sentir comment le dos et la poitrine bougent, les genoux, les pieds et les jambes. Donc il y a une présence dans le corps, et aussi l'environnement... Je suis assise sur [elle frappe le plancher] comme l'oiseau qui marche là dehors. Il y a tellement d'égalité là-dedans. Et de vie [elle frappe le plancher]. Je fais partie d'un tout connecté. Il y a une telle impression d'appartenir, juste parce que [elle rit], juste parce que c'est là, oui. Et quand l'attention part dans les pensées, c'est comme si... il y a un sentiment de séparation, comme ça [geste]. Je pense que c'est comme cette membrane [geste] dont je parlais tout à l'heure, elle est fermée, vous êtes ici et le monde est là-bas, oui. Et c'est simplement extrêmement libérateur de retourner à la respiration et au corps. Et sentir cela rend les choses plus vraies." (Helen)

\section{Troisième partie. Discussion}

Cette étude nous a permis de recueillir des descriptions fines de la dynamique de deux processus, la dérive attentionnelle et l'émergence d'une pensée, et d'identifier dans ces deux expériences un ensemble de catégories descriptives indépendantes de leur contenu, en d'autres termes une ébauche de structure dynamique de ces expériences. Nous allons maintenant discuter du statut de ces résultats, des directions de recherche qu'ils ouvrent, et enfin de l'intérêt de ces descriptions pour le méditant et pour l'instructeur de méditation.

\subsection{Statut de ces résultats}

Les analyses que nous avons présentées dans la section précédente sont loin d'épuiser le contenu des entretiens, qui ont été seulement partiellement consacrés à la description d'épisodes de dérive attentionnelle et à celle de l'émergence de pensées. Et ces descriptions sont elles-mêmes loin d'épuiser ces deux types d'expérience. Ces descriptions et leurs analyses donnent donc des pistes de recherches futures. Des chercheurs formés à la micro-phénoménologie pourraient considérer les catégories expérientielles que nous avons identifiées :

- comme des hypothèses à confirmer, affiner ou invalider par de nouveaux entretiens et de nouvelles analyses, dans le contexte de protocoles expérientiels identiques ou différents;

- comme des guides pour de futurs entretiens. Pour chaque catégorie descriptive que nous avons identifiée, le chercheur pourrait de demander : "Quel protocole expérientiel, quelles questions puis-je utiliser pour recueillir l'information correspondante ?". Par exemple, quelles questions peuvent amener un méditant à décrire sa "position perceptuelle dans la scène virtuelle" ? Comment obtenir une description plus précise des gestes intérieurs consistant à "revenir aux narines", à "relâcher la respiration", ou à "descendre dans son corps" ?

- comme des heuristiques permettant de découvrir de nouvelles dimensions à explorer. Les manques mêmes de nos analyses suggèrent des investigations complémentaires pour les compléter. Par exemple il serait possible de produire des descriptions plus fines du processus d'émergence de scènes virtuelles, incluant l'ordre d'apparition des modalités sensorielles, leurs degrés de conscience respectifs, les micro-gestes qui entretiennent la scène, les transitions d'une scène à l'autre, mais aussi la transition entre l'émergence d'une pensée sous la forme d'une minuscule crispation et l'absorption de l'attention dans une scène virtuelle élaborée. Il serait aussi possible d'explorer plus finement le processus inverse de disparition de la scène et la "trace" qu'elle laisse : à quoi ressemble la "trace" laissée par une scène virtuelle ? A-t-elle une forme sensorielle ? Pendant combien de temps persiste-t-elle et peut-elle être "rafraichie" et "réactivée" ?

Dans les descriptions que nous avons recueillies, dès que les prémisses d'une pensée apparaissent sous la forme d'une minuscule "impulsion", au moins dans certains cas, une tension 
subtile a pour effet de produire un sentiment de déconnection entre la tête et le corps ou un sentiment d'inconfort (un "engourdissement"). Quand au cours d'un épisode de dérive attentionnelle la pensée naissante se transforme en scène virtuelle, cette perte de conscience corporelle peut s'intensifier pour aboutir à une perte de conscience totale des sensations corporelles. Nos descriptions suggèrent que cette perte pourrait susciter une sorte de rigidification et de coupure non seulement entre la tête et le corps, mais entre l'espace "interne" et l'espace "externe", entre le sujet et l'environnement. Inversement, le retour aux sensations actuelles permet à la vie, à la chaleur de circuler à nouveau. Il suscite une réunification de l'esprit et du corps, de soi et du monde, qui crée un sentiment de profond soulagement et de libération.

Il nous semble que ces descriptions offrent une piste intéressante pour expliquer le mal-être souvent associé à la dérive attentionnelle (Killingsworth \& Gilbert 2010), comme le possible effet thérapeutique de la méditation (e.g., Farb et al. 2015). Nous faisons l'hypothèse que l'inconfort généré par la dérive n'est pas (seulement) dû au contenu (agréable ou désagréable) de la scène virtuelle, mais à la perte même de contact avec l'intimité de l'expérience, notamment corporelle. L'effet thérapeutique de la méditation s'expliquerait non par l'obtention d'un certain contenu d'expérience, mais par le processus même de reprise de contact avec l'expérience, quel que soit son contenu. Dans les deux cas, l'effet serait du non au contenu de l'expérience (pensée ou scène virtuelle), mais aux caractéristiques structurelles du processus même de prise (ou perte) de conscience.

Une description plus approfondie du processus de perte de contact avec l'expérience pourrait également éclairer le processus qui sous-tend la notion Bouddhiste d'avidyā, "ignorance" or "nescience", qui nous empêche de "voir les choses comme elles sont". Nous pourrions notamment investiguer le processus par lequel l'avidyā crée et maintient l'illusion co-émergente d'un sujet permanent désirant s'approprier des objets solides, considérée dans la tradition Bouddhiste comme la racine même de la souffrance, et le processus inverse d'évanouissement de cette illusion, qui mène à la libération de la souffrance.

\subsection{Utilité de ces descriptions pour les pratiquants et les enseignants de méditation}

Selon les "post-entretiens" conduits avec les pratiquants, les entretiens microphénoménologiques ont été intéressants et utiles, parce qu'ils leur ont permis d'affiner la conscience de ce qui se passe et de ce qu'ils font réellement pendant qu'ils pratiquent. C'est comme "faire un voyage de découverte", dit Helen. Certains méditants expriment aussi un doute sur la capacité des mots à exprimer l'expérience avec justesse : les mots peuvent ne pas correspondre exactement à l'expérience, ou interrompre le flux de l'expérience. Tous s'accordent néanmoins sur le fait que l'entretien "déplie" l'expérience :

"J'ai la sensation d'arriver à déployer l'expérience, comme une feuille chiffonnée qu'on arrive à déplier avec cette enquête." (Elisa)

"Avant l'entretien, je pensais que ma méditation de ce matin n'était pas une grande méditation... je me suis dit que ce n'était pas intéressant, qu'il n'y avait rien. Mais pendant l'entretien, je me suis aperçue qu'il y a de la matière pour une vie entière... Merci !" (Linda)

Ce "déploiement" est dû au fait que les pratiquants prennent conscience d'éléments de leur expérience précédemment non remarqués :

"Le travail d'explicitation a attiré mon attention sur ces petits moments que sinon je n'aurais peut-être pas remarqués. Cela affine ma conscience du processus." (Joëlle)

"Cela donne une plus grande vigilance. Oui. Une plus grande conscience des transitions. De ce qui se passe. Plus de subtilité. [. . .] Cela met en évidence les différentes composantes. Et cela donne une richesse qui en fait est là tout le temps, mais cela la rend plus accessible." (Helen) 
Ces éléments non remarqués peuvent être des sensations subtiles, ou la localisation de ces sensations :

"J'ai pris conscience que l'émergence des pensées est associée à un petit "élan" au niveau de la poitrine. C'est intéressant pour moi parce que je ne l'avais pas remarqué." (Lise)

"Ce sentiment de vouloir aller ailleurs que là où je suis... J'ai été surprise et intriguée par ce sentiment... 'oh, cela vient du somment de ma tête ! Sur la droite et non au centre'." (Anna)

"Je n'avais pas vraiment conscience que le resserrement était autour de la gorge. C'est quelque chose dont j'ai pris conscience pendant l'entretien. Parce que j'aurais pensé que c'était encore un resserrement dans la tête. Mais non. C'est dans la gorge." (Anna)

La plupart des pratiquants interviewés ont signalé qu'ils avaient pris conscience non seulement de sensations, mais aussi de micro-gestes subtils, en d'autres termes de ce qu'ils font réellement pendant leur pratique, par exemple :

- des micro-gestes impliqués dans le processus d'émergence et d'entretien des pensées : depuis la tension infime vers la première impulsion d'une pensée émergente, jusqu'aux gestes qui génèrent et alimentent une scène virtuelle dans laquelle le pratiquant s'absorbe;

- des micro-gestes impliqués dans le processus d'évanouissement des pensées, comme le relâchement de la tension vers une pensée émergente, ou le geste de "descendre dans son corps".

Par exemple, dans l'extrait suivant, l'entretien permet au pratiquant d'affiner sa conscience de ce qu'il fait réellement lorsqu'il "ramène l'attention au niveau des narines".

"Je suis en train de réaliser qu'en fait, quand je ramène mon attention aux narines, je ne déplace pas (mon attention). Il n'y a pas de déplacement d'un point à un autre, en tout cas pas là dans mon expérience. Ce n'est pas : il n'y a pas conscience du nez, et puis d'un seul coup il y a conscience du nez. La conscience du nez, de l'air, elle est déjà là, mais ce que je fais c'est la renforcer, l'intensifier. Il y a comme un mouvement, mais je ne trouve pas de point de départ à ce mouvement. Le point d'arrivée, c'est l'intensification. Mais pour l'instant je n'ai pas trouvé de point de départ. Donc quand je déplace mon attention, il y a un renforcement, une intensification de la densité. Et il y a une action pour le faire. (...) Grâce à l'entretien, j'ai réalisé qu'il n'y a pas de déplacement de l'attention. C'est presque une découverte pour moi. Ce n'est pas une découverte qui me surprend, parce que c'est mon expérience. Mais à première vue j'aurais dit que je déplace mon attention. Mais ce n'est pas vrai." (Paul)

Ce sont les procédés spécifiques de l'entretien qui permettent aux sujets de discriminer ces sensations et gestes :

1) la précision des questions, qui attirent l'attention du pratiquant vers des dimensions et moments particuliers de son expérience.

- Et qu'est-ce qui vous permet de vous en rendre compte?

- Que vous me demandiez. Que vous me demandiez ce qui arrive juste avant. Que vous reveniez à cela. Que se passe-t-il juste avant? Que vous pointiez une fraction de seconde et me demandiez: que se passet-il là? Et alors l'attention doit y aller et sentir ce qui se passe là. Qu'est-ce qui se passe là ? Qu'est-ce qui se passe là ? Et qu'est-ce que je fais, qu'est-ce que je fais ? Qu'est-ce que ça fait dans le corps ? Qu'est-ce qui arrive à l'attention là ? À quoi ça ressemble ? Que vous demandiez, que vous pointiez un point précis, c'est suffisant. Peut-être que sinon je ne l'aurais simplement pas remarqué. (Helen)

2) le pouvoir du processus d'évocation:

"C'était en rapport avec cet effet de zoom que j'ai senti que ça fonctionnait effectivement. C'était en rapport avec certains moments où le fait de le ressentir à nouveau a rétabli cet état de manière si précise que j'ai pu me souvenir de plus que je n'aurais pu le faire quelques instant plus tôt. C'était vraiment intéressant." (Martin)

3) et l'effort même pour trouver les mots :

"Je trouve que c'est intéressant de remarquer à quel point la pratique peut être là et être aiguisée au moment où on y met des mots, presque comme une conséquence de la mettre en mots." (Marcus) 
"Une chose que je constate avec l'explicitation c'est qu'elle aiguise votre conscience, la clarté sur ce qui se passe réellement. Et l'aiguisement réside dans le travail qui consiste à trouver des mots. Trouver des mots et ensuite découvrir, du moins parfois, que ce n'était pas assez précis et ensuite chercher à affiner encore." (Helga)

Ces témoignages montrent que ce qui est important dans l'entretien micro-phénoménologique est moins la description en tant que résultat que l'acte même de décrire, c'est-à-dire les gestes intérieurs de discrimination que le pratiquant réalise, comme "effets perlocutoires" (Austin, 1962) des questions et relances de l'intervieweur.

Dans cette perspective, les descriptions verbales ne sont autres que des "poignées" permettant au pratiquant de discriminer des aspects subtils de son expérience qui auraient pu s'évanouir sans leur aide. Ils n'expriment pas nécessairement l'expérience, ne la reflètent pas, mais l'indiquent ${ }^{5}$.

Malgré leur insuffisance, les mots ont aussi le pouvoir de susciter un acte de reconnaissance chez l'interlocuteur ou le lecteur. Dans l'extrait suivant, un intervieweur observe que le mot utilisé par un pratiquant lui a permis de reconnaître dans sa propre expérience méditative un élément qu'elle n'avait pas remarqué auparavant :

"L'entretien d'hier avec Paul quand il a décrit cette 'impulsion' m'a permis d'en prendre conscience, même si elle m'était déjà familière. Le mot 'impulsion' me convient bien. J'ai l'impression de reconnaître l'expérience qu'il indique." (Lise)

La reconnaissance de l'expérience indiquée par le mot, et l'adoption de ce mot par le chercheur, marque le début d'un possible accord intersubjectif sur ce mot pour désigner ce mouvement subtil. En d'autres termes, la fonction indicielle des mots n'empêche pas la création d'un vocabulaire partagé et spécialisé pour communiquer à propos de l'expérience méditative.

"Entre personnes qui ont vécu des expériences, alors les mots... c'est un peu comme les poètes. Les poètes utilisent des mots, mais les mots des poètes pointent vers quelque chose bien au-delà des mots. Il y a une résonance de la parole, qui pointe vers autre chose. Donc, pour arriver à faire comprendre à l'autre ce vers quoi on veut pointer, on utilise des mots, sachant que les mots ne sont que des directions qu'on donne." (Alain)

Même les méditants expérimentés ont reconnu que les entretiens les avaient aidés à prendre conscience d'éléments non remarqués. Ce qui répond à l'une des questions que nous nous posions au début du projet : le but de la méditation est de "voir les choses comme elles sont", de développer la conscience de son expérience, la présence à son expérience; dans ces conditions, en quoi les méthodes micro-phénoménologiques, qui visent aussi à développer la conscience de l'expérience, peuvent-elles être utiles à des pratiquants ? Notre étude montre que même les pratiquants avancés, ayant développé une attention très fine à leur expérience, ne sont pas nécessairement pleinement conscients de toutes ses dimensions et de tous les micro-gestes qu'ils réalisent pour s'y rendre présents. Le fait d'être encouragés à les décrire très précisément et aidés à le faire, leur permet d'en acquérir une conscience plus fine, et peut-être de les effectuer de manière plus précise. Autrement dit les méthodes micro-phénoménologiques pourraient encourager le processus de discrimination visé par exemple par vipaśyanā.

Certains pratiquants interviewés craignent aussi que l'entretien ne crée une tension supplémentaire. Le fait de méditer en sachant qu'on sera interviewé pourrait créer un effort pour mieux observer et se souvenir. Mais comme l'un d'entre eux l'a remarqué, cette tension est probablement toujours présente dans la pratique, et la situation d'entretien permet seulement d'en prendre mieux conscience.

\footnotetext{
${ }^{5}$ Comme l'a écrit Heidegger, "les concepts phénoménologiques ne peuvent communiquer entièrement leur contenu, mais seulement l'indiquer." (Zahavi 2003, p. 173).
} 
De plus, une fois qu'un entretien a permis au pratiquant de reconnaître des éléments auparavant non remarqués de son expérience et de sa pratique, la conscience de ces éléments persiste dans les séances de méditation ultérieures. Il n'est donc pas nécessaire de maintenir l'effort discriminant dans toutes les sessions de méditation. Par exemple, si un entretien a permis au pratiquant de prendre conscience d'une petite "impulsion" au niveau de la poitrine juste avant l'émergence d'une pensée, il pourra la reconnaître dans les sessions futures sans avoir besoin de mobiliser un effort pour l'identifier. L'entretien a un effet d'apprentissage : la conscience aiguisée par l'entretien apporte plus de clarté et de précision dans les méditations ultérieures et même en dehors des sessions de méditation.

"C'est vrai que ça a eu une influence sur ma méditation. Ça a provoqué, chez moi, quelque chose qui fait que je suis davantage concentré pendant ma méditation. Depuis la dernière fois, c'est clair et net que je décortique davantage ce qui se passe, je suis plus précis." (Michel)

"Cela accentue la clarté de la conscience. C'est comme si, il est clair que - l'image est peut-être bête, mais il est clair que le miroir a été poli." (Marcus)

"En fait, ça me plaît beaucoup... parce que, même cette semaine (entre les deux entretiens), ça me rend attentif à ce qui se passe en moi. (Alain, 45 years of practice)

Les instructeurs de méditation qui ont été formés à l'entretien micro-phénoménologique attestent que celui-ci leur est aussi utile dans le cadre de leur enseignement. D'une part, une conscience affinée de leur propre pratique les aide à affiner leurs instructions de méditation. D'autre part, mener des entretiens avec leurs étudiants les aide à se faire une meilleure idée de la manière dont ceux-ci pratiquent, et donc à améliorer leur enseignement. Ils développent une palette d'instructions plus riche et plus précisément adaptée aux individus.

\section{Conclusion}

Cette étude pilote nous a permis de recueillir des descriptions fines d'expériences méditatives, de commencer à identifier leurs paramètres et à dégager leur structure. Les hypothèses qui émergent de ce travail pourront être testées par des travaux de recherche futurs grâce à la création de protocoles expérientiels appropriés.

Notre étude montre que l'expérience méditative est un objet de recherche à part entière, qui présente un intérêt inestimable pour 1) comprendre les processus impliqués dans la méditation; 2) comprendre leurs effets, notamment leurs effets thérapeutiques; 3) perfectionner l'enseignement de la méditation; 4) affiner notre compréhension des processus concrets qui sous-tendent certains concepts de l'épistémologie Bouddhiste. De plus, comme la méditation ne vise pas à cultiver des expériences extraordinaires, mais à entraîner l'esprit à prendre conscience des phénomènes tels qu'ils sont, son investigation micro-phénoménologique pourrait éclairer certaines questions épineuses dans les études contemporaines de la conscience, comme l'existence d'un soi "minimal". Une description fine des microprocessus qui créent l'illusion d'un soi permanent face à un monde solide et la scission dualiste entre un sujet voyant et un objet vu, pourrait aussi éclairer le "problème difficile" de la conscience.

Cette investigation micro-phénoménologique n'est pas un simple détour heuristique destiné à fournir des clés pour interpréter l'activité cérébrale, aussi intéressant que cela puisse être pour comprendre les processus méditatifs. En vérité, une meilleure connaissance de la structure expérientielle des processus méditatifs est un pré-requis pour rechercher et interpréter ses éventuels corrélats neurophysiologiques. L'expérience étant première (Bitbol, 2014), son investigation micro-phénoménologique disciplinée en procure une compréhension irremplaçable et irréductible. 


\section{Bibliographie}

Allen, M., Dietz, M. Blair, K., van Beek, M., Rees, G., Vestergaard-Poulsen, P., Lutz, A. Roepstorff, A. (2012). Cognitive-affective neural plasticity following active-controlled mindfulness intervention. Journal of Neuroscience 32 (44): 15601-15610.

Andrews-Hanna JR, Kaiser RH, Turner AE, Reineberg AE, Godinez D, et al. (2013). A penny for your thoughts: dimensions of self-generated thought content and relationships with individual differences in emotional wellbeing. Front. Psychol. 4:900.

Ataria Y. (2014). Where Do We End and where does the World Begin? The Case of Insight Meditation. Philosophical Psychology, 1-19, doi: 10.1080/09515089.2014.969801.

Ataria Y. (2015). Consciousness and Cognition

Austin, J. L. (1962), How to Do Things with Words, Oxford: Oxford University Press

Bitbol M. (2014). La conscience a-t-elle une origine. Paris : Flammarion.

Bitbol M., Petitmengin C. (2013). A defense of Introspection from Within. Constructivist Foundations 8 (3): 269-279

Bitbol M., Petitmengin C. (2016). Neurophenomenology and the elicitation interview. in: M. Velmans (ed.). The Blackwell Companion to Consciousness (2nd edition). Wiley \& Sons.

Brefczynski-Lewis, J. A., Lutz, A., Schaefer, H. S., Levinson, D. B., \& Davidson, R. J. (2007). Neural correlates of attentional expertise in long-term meditation practitioners. Proceedings of the National Academy of Sciences, 104, 11483.11488.

Brewer, J. A., Worhunsky, P. D., Gray, J. R., Tang, Y. Y., Weber, J., \& Kober, H. (2011). Meditation experience is associated with differences in default mode network activity and connectivity. Proceedings of the National Academy of Sciences of the United States of America, 108, 20254-20259.

Desbordes, G., Negi, L. T., Pace, T. W. W., Wallace, B. A., Raison, C. L., \& Schwartz, E. L. (2012). Effects of mindful-attention and compassion meditation training on amygdala response to emotional stimuli in an ordinary, non-meditative state. Frontiers in Human Neuroscience, 6, 292. http://dx.doi.org/10.3389/fnhum.2012.00292

Farb, N. A., Segal, Z. V., Mayberg, H., Bean, J., McKeon, D., Fatima, Z.,\& Anderson, A. K. (2007). Attending to the present: Mindfulness meditation reveals distinct neural modes of self-reference. Social Cognitive and Affective Neuroscience, 2, 313-322.

Farb, N., Daubenmier, J., Price, C.J., Gard, T., Kerr, C., Dunn, B.D., Klein, A.C., Paulus, M.P. \& Mehling, W.E. (2015) Interoception, contemplative practice, and health. Frontiers in Psychology, 6:763. Doi: 10.3389/fpsyg.2015.00763

Fox, K.C.R., Zakarauska, P., Dixon, M., Ellamil, M., Thompson, E. \& Christoff, K. (2012). Meditation Experience Predicts Introspective Accuracy. PLoS ONE 7(9):e45370.

Galera C, Orriols L, M'Bailara K, Laborey M, Contrand B, et al. (2012). Mind wandering and driving: responsibility case-control study. BMJ 345:e8105.

Germano, D. and Hillis, G. A. (2005). Tibetan Buddhist Meditation. Encyclopedia of Religion, (2nd edition). Chicago: Macmillan. 1284-1290.

Grant, J. A., Courtemanche, J., Duerden, E. G., Duncan, G., \& Rainville, P. (2010). Cortical thickness and pain sensitivity in Zen meditators. Emotion, 10, 43-53.

Grossman, P., Tiefenthaler-Gilmer, U., Raysz, A., \& Kesper, U. (2007). Mindfulness training as an intervention for fibromyalgia: Evidence of postintervention and 3-year follow-up benefits in well-being. Psychotherapy and Psychosomatics, 76, 226-233. http://dx.doi.org/10.1159/000101501

Henry, M. (2000). Incarnation. Une philosophie de la chair. Paris: Editions du Seuil

Husserl, E. (2002). Zur phänomenologischen Reduktion. Texte aus dem Nachlass (1926-1935). Sebastian Luft (ed.). Dordrecht: Kluwer

Holzel, B.K., Carmody, J., Vangel, M., Congleton, C., Yerramsetti, S.M., Gard, T., Lazar, S.W., (2011). Mindfulness practice leads to increases in regional brain gray matter density. Psychiatry Res. 191 (1), 36-43.

Jensen, C. G., Vangkilde, S., Frokjaer, V., \& Hasselbalch, S. G. (2012). Mindfulness training affects 
attention-Or is it attentional effort? Journal of Experimental Psychology: General, 141, 106-123. http://dx.doi.org/10.1037/a0024931

Khalsa, S.S., Rudrauf, D., Damasio, A.R., Davidson, R.J., Lutz, A., \& Tranel, D. (2008). Interoceptive awareness in experienced meditators. Psychophysiology, 45, 671-677.

Killingsworth M. \& Gilbert D. (2010). A wandering mind is an unhappy mind. Science 33 (6006).

Kuyken, W., Hayes R., Barrett B., Byng R., Dalgleish T., Kessler D., Lewis G., Watkins E., Brejcha C., Cardy J., Causley A., Cowderoy S., Evans A., Gradinger F., Kaur S., Lanham P., Morant N., Richards J., Shah P., Sutton H., Vicary R., Weaver A., Wilks J., Williams M., Taylor RS., Byford S. (2015) Effectiveness and cost-effectiveness of mindfulness-based cognitive therapy compared with maintenance antidepressant treatment in the prevention of depressive relapse or recurrence (PREVENT): a randomised controlled trial. Lancet, 386, 63-73.

Lachaux J.-P. (2011), "If no control, then what? Making sense of 'neural noise' in human brain mapping experiments using first-person reports", Journal of Consciousness Studies, 18, 162-166.

Lazar, S. W., Kerr, C., Wasserman, R., et al. (2005). Meditation experience is associated with increased cortical thickness. Neuroreport, 16, 1893-1897.

van Leeuwen, S., Singer, W., \& Melloni, L. (2012). Meditation increases the depth of information processing and improves the allocation of attention in space. Frontiers in Human Neuroscience, 6, 133. http://dx.doi.org/10.3389/fnhum.2012.00133

Lutz, A., Dunne, J., \& Davidson, R.J. (2007). Meditation and the Neuroscience of Consciousness: An introduction. In: P. Zelazo, M. Moscovitch and E. Thompson. Cambridge Handbook of Consciousness. Cambridge: Cambridge University Press, 499-552.

Lutz, A., Brefczynski-Lewis, J. A., Johnstone, T., \& Davidson, R. J. (2008). Voluntary regulation of the neural circuitry of emotion by compassion meditation: Effects of expertise. PLoS One, 3(3), e1897.

Lutz, A., McFarlin D.R., Perlman D.M., Salomons T.V., Davidson R.J. (2013). Altered anterior insula activation during anticipation and experience of painful stimuli in expert meditators. Neuroimage (64C), 538-46.

Lutz, A., Jha, A.P., Dunne, J.D., Saron, C.D. (2015). "Investigating the Phenomenological Matrix of Mindfulness-related Practices from a Neurocognitive Perspective." American Psychologist 70 (7): 632658 .

Mooneyham, B.W. \& Schooler, J.W. (2013). The costs and benefits of mind-wandering: A review. Canadian Journal of Experimental Psychology, 67(1), 11-18.

Mrazek, M. D., Franklin, M. S., Phillips, D. T., Baird, B., \& Schooler, J. W. (2013). Mindfulness training improves working memory capacity and GRE performance while reducing mind wandering. Psychological Science, 24, 776-781. http://dx.doi.org/10.1177/0956797612459659

Petitmengin, C. (1999). The Intuitive Experience. In: F.Varela and J. Shear, The View from Within. First-person approaches to the study of consciousness. Exeter: Imprint Academic, 43-77.

Petitmengin, C. (2006). Describing one's subjective experience in the second person: An interview method for the science of consciousness. Phenomenology and the Cognitive Science, 5, 229-269.

Petitmengin C. 2007. "Towards the source of thoughts. The gestural and transmodal dimension of lived experience." Journal of Consciousness Studies, 14: 54-82.

Petitmengin C. (2010). A neuro-phenomenological study of epileptic seizure anticipation. In Daniel Schmicking and Shaun Gallagher (eds.), Handbook of Phenomenology and Cognitive Sciences, Berlin, Heidelberg, New York: Springer, 471-499.

Petitmengin C., Baulac M., \& Navarro V. 2006. "Seizure anticipation: are neurophenomenological approaches able to detect preictal symptoms?" Epilepsy and Behavior, 9: 298-306.

Petitmengin C., Remillieux A., Cahour C., and Carter-Thomas S. 2013. "A gap in Nisbett and Wilson's findings? A first-person access to our cognitive processes". Consciousness and Cognition, 22: 654-669. doi:10.1016/j.concog. 2013.02.004

Petitmengin C. \& Bitbol M. (2009). The Validity of First-Person Descriptions as Authenticity and Coherence. Journal of Consciousness Studies, 16, 363-404. 
Petitmengin C, Remillieux A., Valenzuela C., Analyzing first-person descriptions: A data analysis method for the science of consciousness. Submitted.

Philippot P., Segal Z. (2009). Mindfulness Based Psychological Interventions. Developing Emotional Awareness for Better Being. In: C. Petitmengin (Ed.). Ten Years of Viewing from Within. The Legacy of Francisco Varela. Exeter: Imprint Academic, 285-306.

Reiner, K., Tibi, L., \& Lipsitz, J. D. (2013). Do mindfulness-based interventions reduce pain intensity? A critical review of the literature. Pain Medicine, 14, 230-242. http://dx.doi.org/10.1111/pme.12006

Remillieux A. (2014). Les coulisses d'une invention. Une description expérientielle du processus d'invention technique. Intellectica 61: 273-310.

Ruby FJM, Smallwood J, Sackur J, Singer T. 2013. Is self-generated thought a means of social problem solving? Front. Psychol. 4:962.

Schooler, J. W., Reichle, E. D., \& Halpern, D. V. (2005). Zoning-out during reading: Evidence for dissociations between experience and meta-consciousness. In D. T. Levin (Ed.), Thinking and seeing: Visual metacognition in adults and children (pp. 203-226). Cambridge, MA: MIT Press.

Schooler JW, Mrazek MD, Franklin MS, Baird B, Mooneyham BW, et al. 2014. The middle way: finding the balance between mindfulness and mind-wandering. In The Psychology of Learning and Motivation, Vol. 60, ed. BH Ross, pp. 1-33. Burlington, MA: Academic

Segal, Z. V., Williams, J. M. G., \& Teasdale, J. D. (2002). Mind-fulness-based cognitive therapy for depression: A new approach to preventing relapse. New York: Guilford Press.

Smallwood, J., \& Schooler, J. W. (2006). The restless mind. Psychological Bulletin, 132, 946-958.

Smallwood J, O'Connor RC, Sudbery MV, Obonsawin M. 2007. Mind-wandering and dysphoria. Cogn.Emot. 21:816-42

Smallwood J, Andrews-Hanna J. 2013. Not all minds that wander are lost: the importance of a balanced perspective on the mind-wandering state. Front. Psychol. 4:441

Slagter, H. A., Lutz, A., Greischar, L. L., Francis, A. D., Nieuwenhuis, S., Davis, J. M., et al. (2007). Mental training affects distribution of limited brain resources. PLoS Biology, 5(6), e138.

Teasdale JD, Segal ZV, Williams JMG, Ridgeway VA, Soulsby JM, Lau MA (2000). Prevention of relapse/recurrence in major depression by mindfulness-based cognitive therapy. J Consult Clin Psychol 68: 615-23.

Tulku Urgyen Rinpoche. (1999, 2000) As It Is. Hongkong and Esby: Rangjung Yeshe Publications

Valenzuela Moguillansky, C., O'Regan, JK. and Petitmengin, C. (2013) Exploring the subjective experience of the "rubber hand" illusion. Frontiers in Human Neurosciences 7:659

Vermersch, P. (1994/2010). L'entretien d'explicitation. Paris: ESF.

Vermersch, P. (2009). Describing the Practice of Introspection. In: C. Petitmengin (Ed.). Ten Years of Viewing from Within. The Legacy of Francisco Varela. Exeter: Imprint Academic, 20-57.

Vermersch, P. (2012). Explicitation et phénoménologie. PUF: Paris.

Yanko MR, Spalek TM. 2013. Driving with the wandering mind: the effect that mind-wandering has on driving performance. Hum. Factors 56:260-69

Zahavi, D. (2003). How to investigate subjectivity: Natorp and Heidegger on Reflection. Continental Philosophy Review, 36 (2):155-76.

Zanesco, A. P., King, B. G., Maclean, K. A., \& Saron, C. D. (2013). Executive control and felt concentrative engagement following intensive meditation training. Frontiers in Human Neuroscience, 7 , 566. http://dx.doi.org/10.3389/fnhum.2013.00566 